\title{
Aeroelastic-Acoustics Simulation of Flight Systems
}

\author{
K. K. Gupta ${ }^{1}$ \\ NASA-DRFC, Edwards, CA \\ S. B. Choi ${ }^{2}$ \\ Advanced Engineering Solutions, Inc., Ormond Beach, FL
}

A. Ibrahim ${ }^{3}$

Norfolk State University, Norfolk, VA 


\section{Introductory remarks}

- Paper describes a numerical FE based aeroelastic-acoustics analysis methodology

- This is followed by solution details of a 2-D airfoil and results correlation with known theoretical solution

- Also presented the 3-D wing case and related results pertaining to vibration, steady and unsteady flow (CFD), aeroelastic and aeroelastic-acoustic simulations

- Further associated solution results are presented for a numerically simulated unsteady pressure data

- Also SPL results from a SOFIA flight sensor data is presented in some detail

- Provides a discussion on implementation of these techniques in an existing FE software suitable for solution of complex, practical problems

- Discussions and concluding remarks 


\section{Introductory remarks}

- Paper describes a numerical FE based aeroelastic-acoustics analysis methodology

- This is followed by solution details of a 2-D airfoil and results correlation with known theoretical solution

- Also presented the 3-D wing case and related results pertaining to vibration, steady and unsteady flow (CFD), aeroelastic and aeroelastic-acoustic simulations

- Further associated solution results are presented for a numerically simulated unsteady pressure data

- Also SPL results from a SOFIA flight sensor data is presented in some detail

- Provides a discussion on implementation of these techniques in an existing FE software suitable for solution of complex, practical problems

- Discussions and concluding remarks 


\section{Numerical Formulation}

- Structural (FE) free vibration matrix equation solving for $\omega$ and $\varphi$

$$
M \ddot{u}+K u=0
$$

- The aerodynamic data are next computed by solving the Navier-Stokes (FE) equation

$$
\frac{\partial \boldsymbol{v}}{\partial t}+\frac{\partial \boldsymbol{f}_{j}}{\partial x_{j}}+\frac{\partial \boldsymbol{g}_{j}}{\partial x_{j}}=\boldsymbol{f}_{b} \quad j=1,2,3
$$

in which

$$
\boldsymbol{v}=\left[\begin{array}{lll}
\rho & \rho u_{i} & \rho E
\end{array}\right]^{T} \quad i=1,2,3
$$

- Vehicle equation of motion is then cast into the frequency domain

$$
\hat{\boldsymbol{M}} \ddot{\boldsymbol{q}}+\hat{\boldsymbol{C}} \dot{\boldsymbol{q}}+\hat{\boldsymbol{K}} \boldsymbol{q}+\boldsymbol{f}_{a}(t)+\boldsymbol{f}_{I}(t)=0
$$

where

$\hat{\boldsymbol{M}}\left(=\boldsymbol{\Phi}^{T} \boldsymbol{M} \boldsymbol{\Phi}\right)$ is the generalized mass matrix and similarly $\hat{\boldsymbol{K}}, \hat{\boldsymbol{C}} ; \boldsymbol{f}_{a}(t)$ is the aerodynamic load vector; $f_{I}(t)$ being the generalized impulse force vector 


\section{Numerical Formulation (cont'd)}

- Generalized impulse force vector $f_{I}(t)$

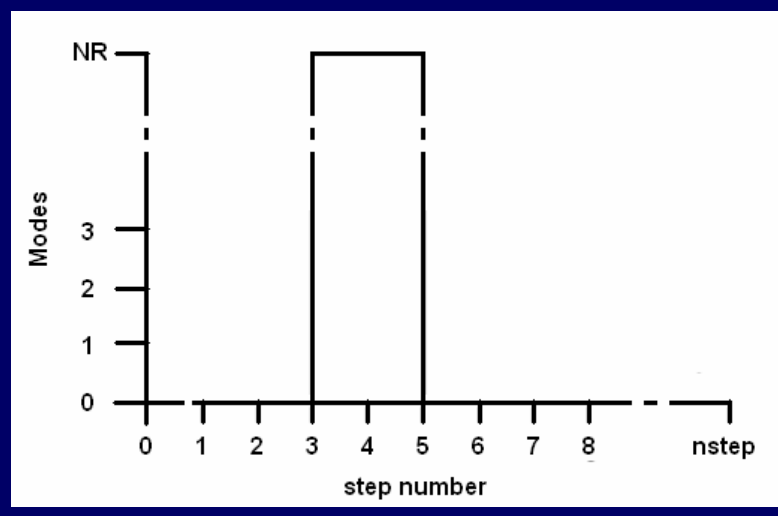

- Earlier, the CFD code analysis results were verified with flight test data

- Hyper-X vehicle and flight data comparison
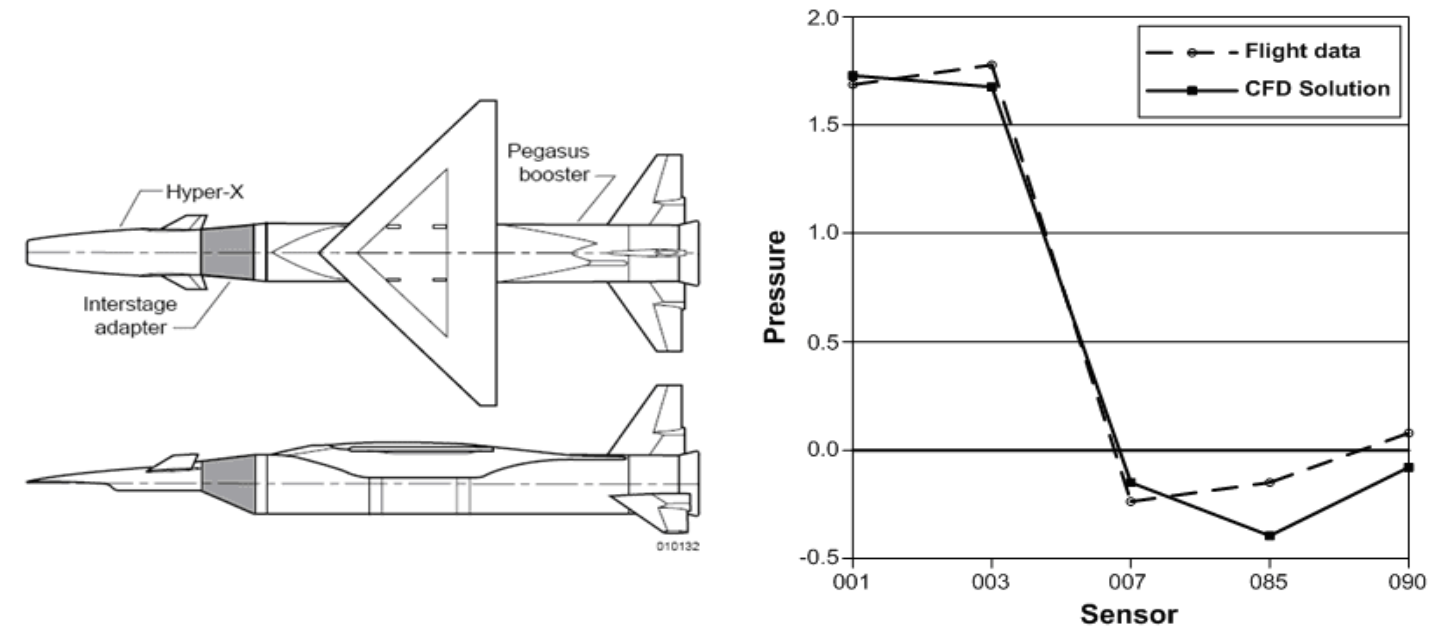


\section{Numerical Formulation (cont'd)}

- Equation (3) may then be cast in a state-space matrix form as

$$
\left[\begin{array}{ll}
\boldsymbol{I} & \mathbf{0} \\
\mathbf{0} & \boldsymbol{I}
\end{array}\right]\left[\begin{array}{l}
\dot{\boldsymbol{q}} \\
\ddot{\boldsymbol{q}}
\end{array}\right]-\left[\begin{array}{cc}
\mathbf{0} & \boldsymbol{I} \\
-\hat{\boldsymbol{M}}^{-1} \hat{\boldsymbol{K}} & -\hat{\boldsymbol{M}}^{-1} \hat{\boldsymbol{C}}
\end{array}\right]\left[\begin{array}{l}
\boldsymbol{q} \\
\dot{\boldsymbol{q}}
\end{array}\right]-\left[\begin{array}{c}
\mathbf{0} \\
-\hat{\boldsymbol{M}}^{-1} \boldsymbol{f}_{a}(t)
\end{array}\right]-\left[\begin{array}{c}
\mathbf{0} \\
-\hat{\boldsymbol{M}}^{-1} \boldsymbol{f}_{I}(t)
\end{array}\right]=\mathbf{0}
$$

or

$$
\dot{\boldsymbol{x}}_{s}(t)=\boldsymbol{A}_{s t} \boldsymbol{x}_{s}(t)+\boldsymbol{B}_{s t} f(t)
$$

where

$$
\boldsymbol{A}_{s t}=\left[\begin{array}{cc}
\mathbf{0} & \boldsymbol{I} \\
-\hat{\boldsymbol{M}}^{-1} \hat{\boldsymbol{K}} & -\hat{\boldsymbol{M}}^{-1} \hat{\boldsymbol{C}}
\end{array}\right], \quad \boldsymbol{B}_{s t}=\left[\begin{array}{c}
\mathbf{0} \\
-\hat{\boldsymbol{M}}^{-1}
\end{array}\right], \quad \boldsymbol{f}(t)=\boldsymbol{f}_{a}(t)+\boldsymbol{f}_{I}(t), \quad \boldsymbol{x}_{s}=\left[\begin{array}{c}
\boldsymbol{q} \\
\dot{\boldsymbol{q}}
\end{array}\right]
$$

and

$$
\boldsymbol{y}_{s}(t)=\boldsymbol{C}_{s t} \boldsymbol{x}_{s}(t)+\boldsymbol{D}_{s t} \boldsymbol{f}(t)
$$

in which $\boldsymbol{C}_{s t}=\boldsymbol{I}$ and $\boldsymbol{D}_{s t}=\mathbf{0}$ 


\section{Numerical Formulation (cont'd)}

- In the presence of sensors, for the most general aeroservoelastic case these equations are converted into zero order hold $(\mathrm{ZOH})$ discrete time equivalent at the $k$-th step:

$$
\begin{aligned}
& \boldsymbol{x}_{s}(k+1)=\boldsymbol{G}_{s} \boldsymbol{x}_{s}(k)+\boldsymbol{H}_{s} \boldsymbol{f}(k) \\
& \boldsymbol{y}_{s}(k+1)=\boldsymbol{C}_{s} \boldsymbol{x}_{s}(k)+\boldsymbol{D}_{s} \boldsymbol{f}(k)
\end{aligned}
$$

in which

$$
\boldsymbol{f}(k)=\boldsymbol{f}_{a}(k)+\boldsymbol{f}_{I}(k)
$$

and

$$
\boldsymbol{G}_{s}=e^{\boldsymbol{A}_{s} \Delta t} \quad \boldsymbol{H}_{s}=\left\lfloor e^{\boldsymbol{A}_{s} \Delta t}-\boldsymbol{I}\right\rfloor\left[\boldsymbol{A}_{s}^{-1} \boldsymbol{B}_{s}\right\rfloor
$$

where $\boldsymbol{A}_{s}$ and $\boldsymbol{B}_{s}$ are $\boldsymbol{A}_{s t}$ and $\boldsymbol{B}_{s t}$ having been modified to include sensors 


\section{Numerical Formulation (cont'd)}

- Coupled aeroelastic (AE) model

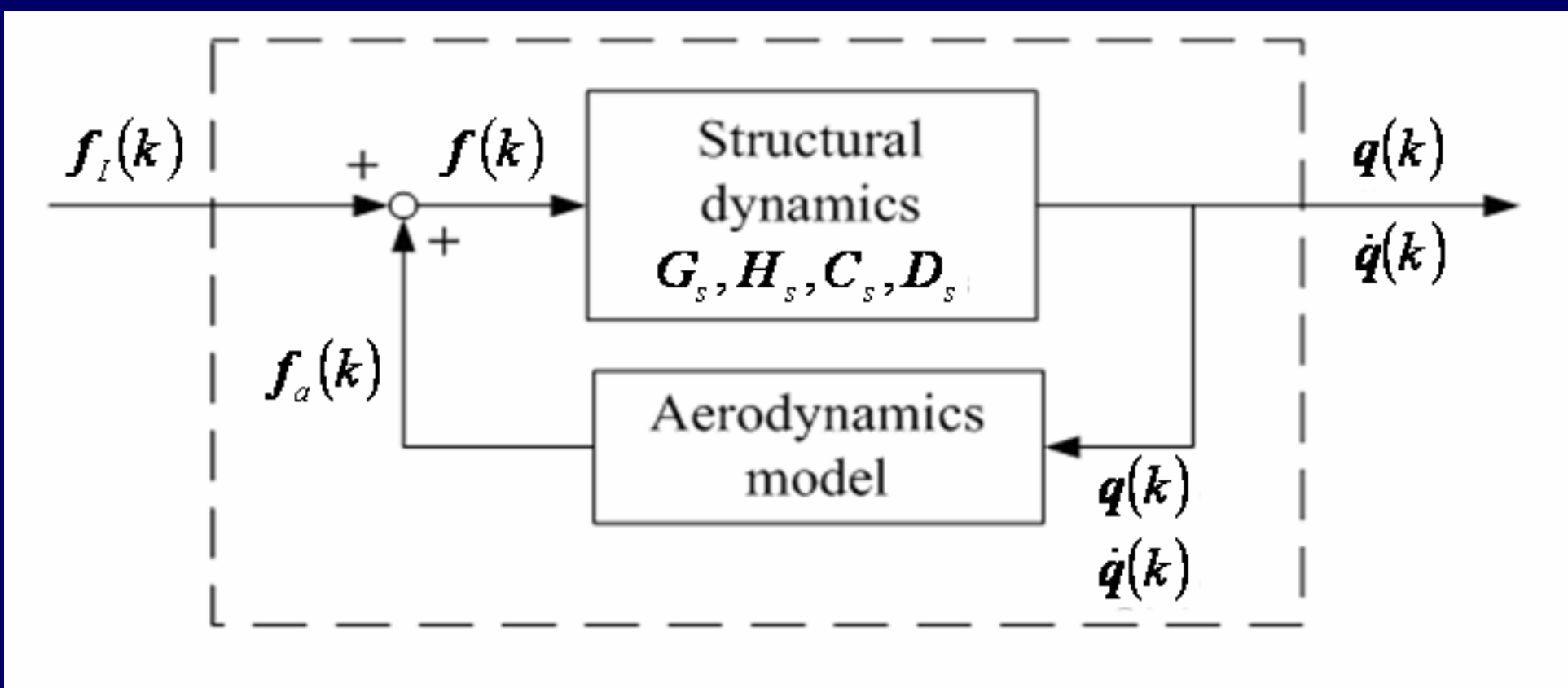




\section{Numerical Formulation (cont'd)}

- Acoustic frequencies are obtained by performing FFT on computed unsteady aerodynamic pressures

- Also the sound pressure level (SPL) for a specified node is computed by first fixing a time band, $t$ and then performing the following calculation using $n$ number of sampling points

a) compute average pressure

$$
P_{\text {avg }}=\left(\sum_{i=1}^{n} P_{i}\right) / n
$$

b) compute the root mean square of pressure

$$
P_{r m s}=\sqrt{\sum_{i=1}^{n}\left(P_{i}-P_{a v g}\right)^{2} / n}
$$

c) compute the SPL

$$
\operatorname{SPL}(d B)=20 \log _{10} \frac{P_{r m s}}{P_{r e f}}
$$

where $P_{r e f}=20 \times 10^{-6} \mathrm{~Pa}$ for air 


\section{Flowchart of aero-elastic-servo-acoustic analysis}

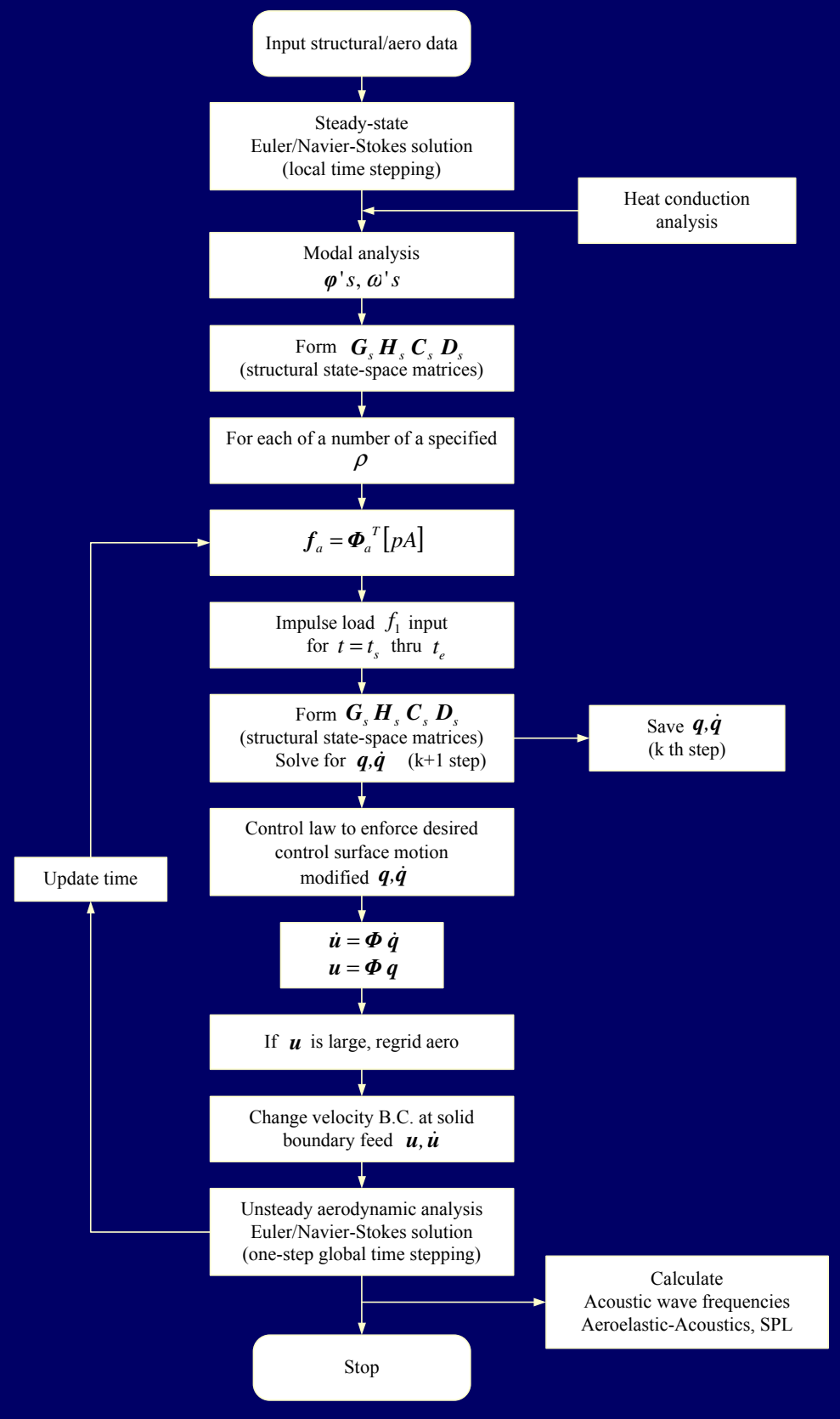




\section{Introductory remarks}

- Paper describes a numerical FE based aeroelastic-acoustics analysis methodology

- This is followed by solution details of a 2-D airfoil and results correlation with known theoretical solution

- Also presented the 3-D wing case and related results pertaining to vibration, steady and unsteady flow (CFD), aeroelastic and aeroelastic-acoustic simulations

- Further associated solution results are presented for a numerically simulated unsteady pressure data

- Also SPL results from a SOFIA flight sensor data is presented in some detail

- Provides a discussion on implementation of these techniques in an existing FE software suitable for solution of complex, practical problems

- Discussions and concluding remarks 


\section{Numerical Example I, 2-D}

- 2-D subsonic NACA 0012 Airfoil

- Flight Condition : Mach Number = 0.3, Angle of Attack = 5-degree

- In an effort to verify the solution accuracy:

- Steady state : Correlation with (i) Smith-Hess panel method (ii) CFD solver

- Unsteady analysis solution is compared with Wagner's suddenly accelerated airfoil problem

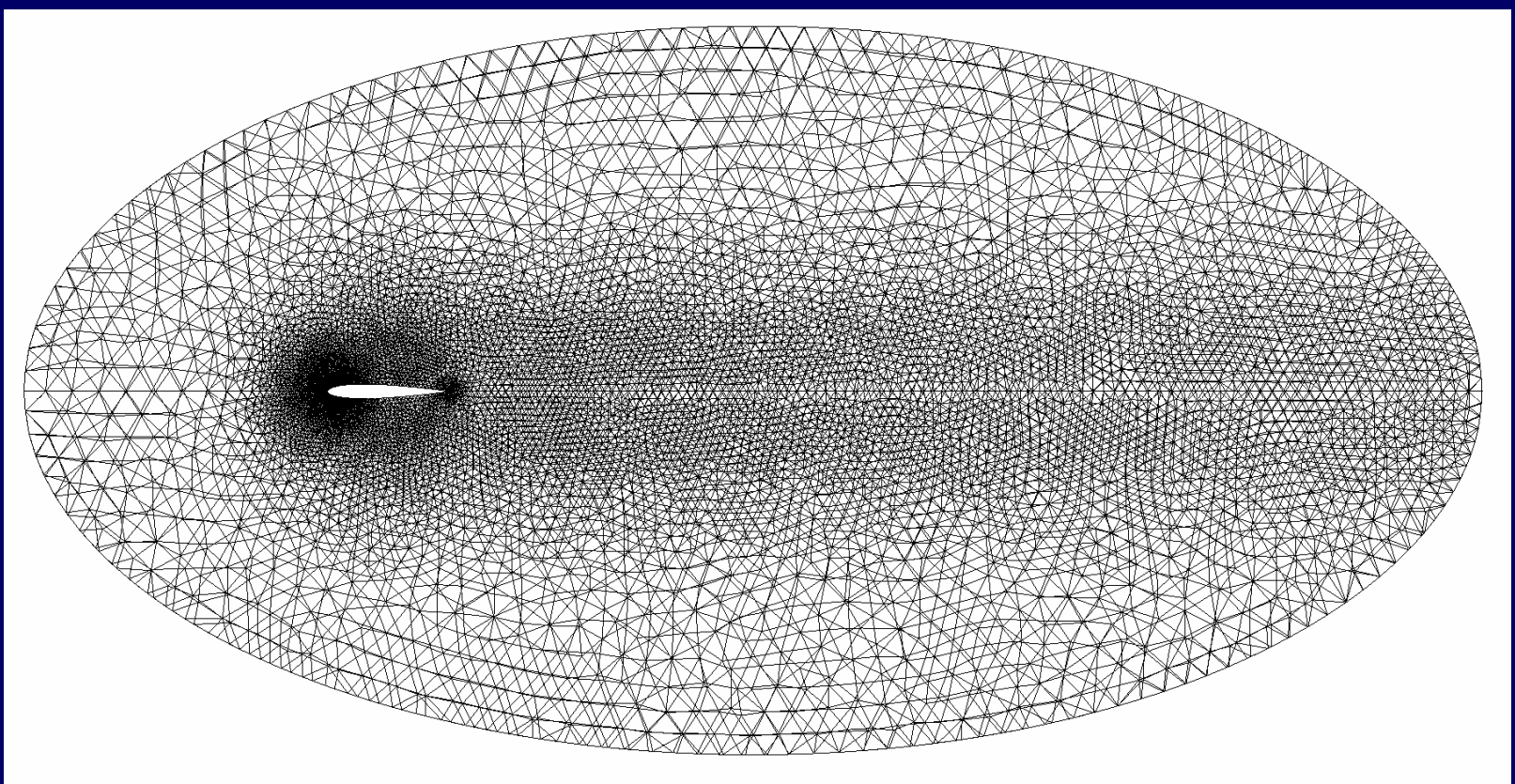




\section{Numerical Example I, 2-D (cont'd)}

- Steady state solutions

1. Coefficient of Pressure (Cp) distribution over solution domain

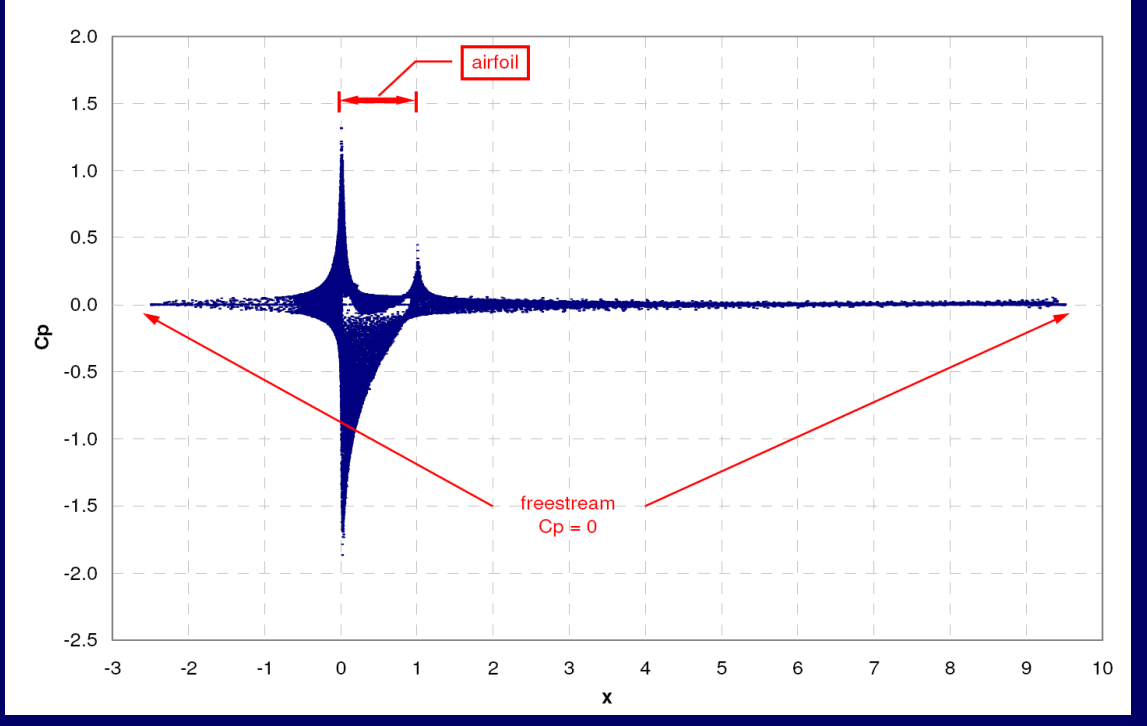

2. Coefficient of Pressure (Cp) distribution comparison on the airfoil

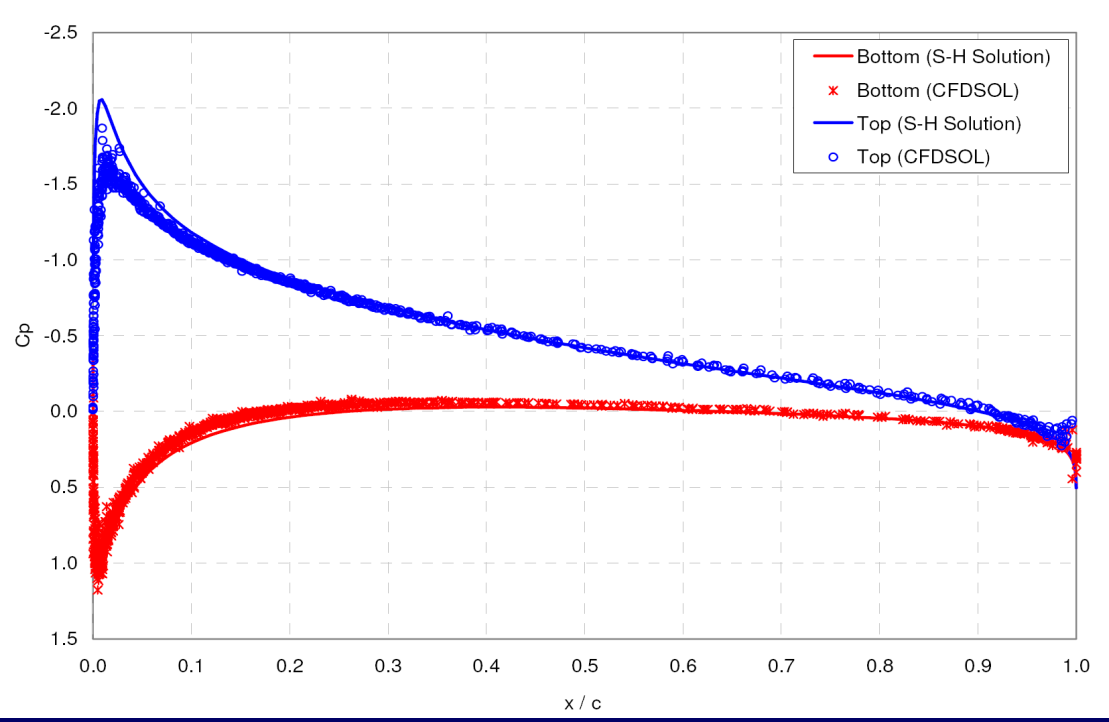




\section{Numerical Example I, 2-D (cont'd)}

- Unsteady analysis solution

- Unsteady Coefficient of Lift (Cl) history comparison

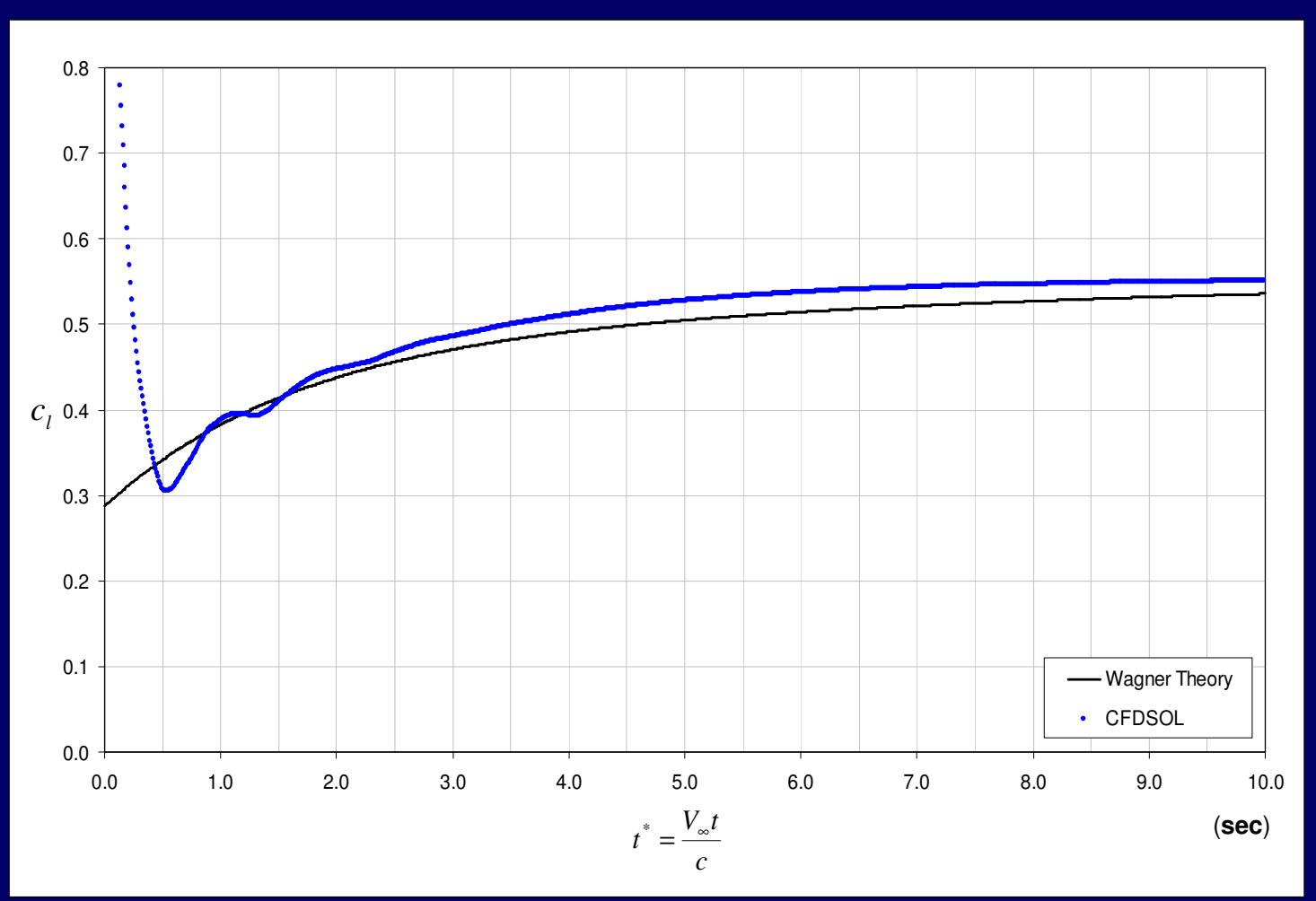




\section{Numerical Example I, 2-D (cont'd)}

- Unsteady analysis solutions

- Coefficient of Pressure (Cp)
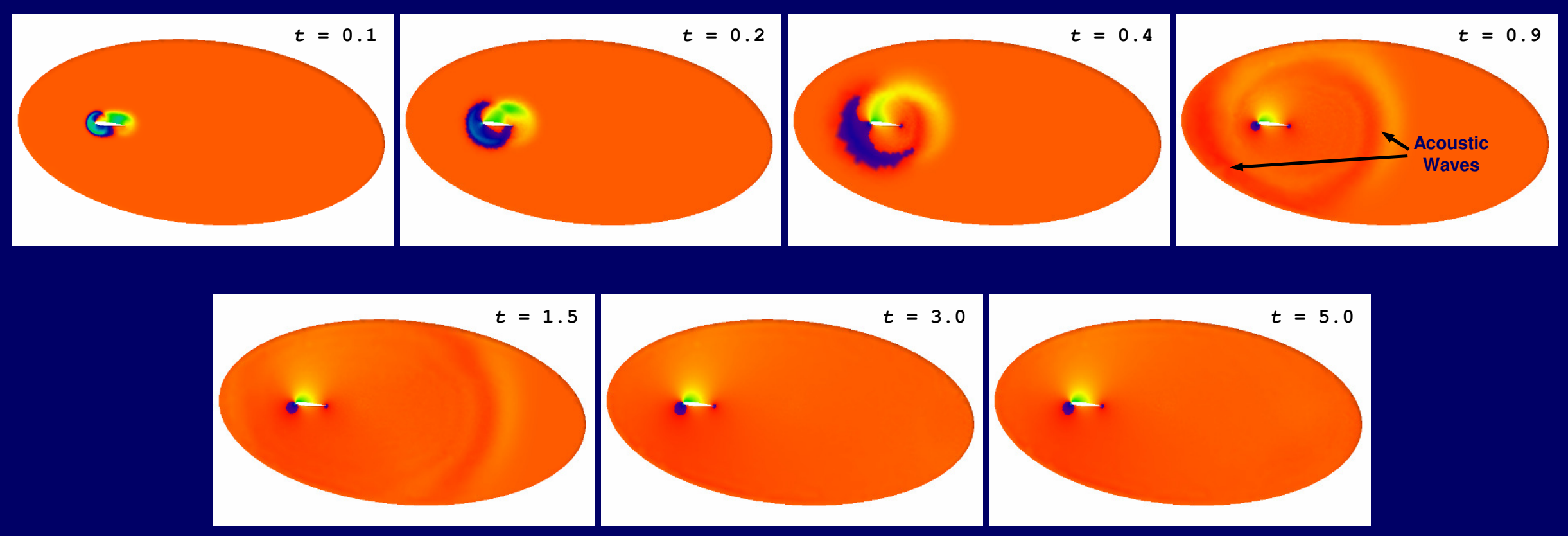


\section{Numerical Example I, 2-D (cont'd)}

- Unsteady analysis solutions

- Velocity
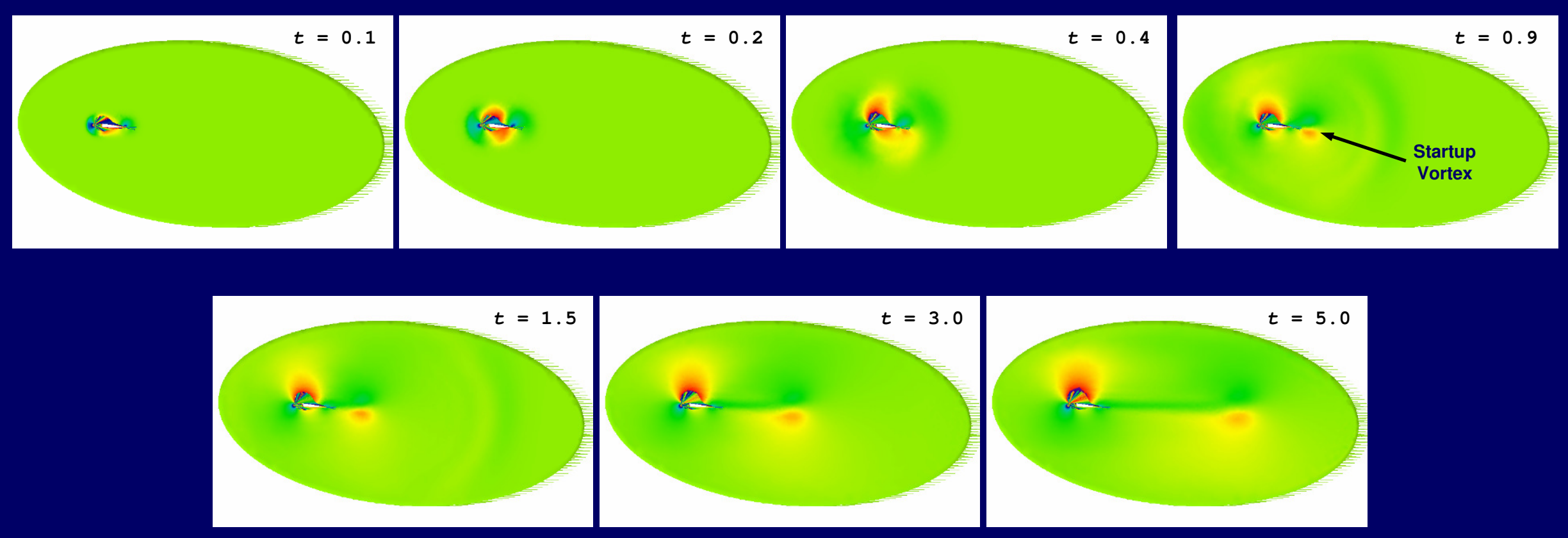

1.5

1.124

0.749

0.375

0.0007 


\section{Introductory remarks}

- Paper describes a numerical FE based aeroelastic-acoustics analysis methodology

- This is followed by solution details of a 2-D airfoil and results correlation with known theoretical solution

- Also presented the 3-D wing case and related results pertaining to vibration, steady and unsteady flow (CFD), aeroelastic and aeroelastic-acoustic simulations

- Further associated solution results are presented for a numerically simulated unsteady pressure data

- Also SPL results from a SOFIA flight sensor data is presented in some detail

- Provides a discussion on implementation of these techniques in an existing FE software suitable for solution of complex, practical problems

- Discussions and concluding remarks 


\section{Numerical Example II, 3-D}

- 3-D cantilever wing with NACA 0012 airfoil

- Flight condition : Mach 0.3 and 0.6, Angle of Attack $=0$ degree

1. Cantilever wing with aeroelastic solution domain

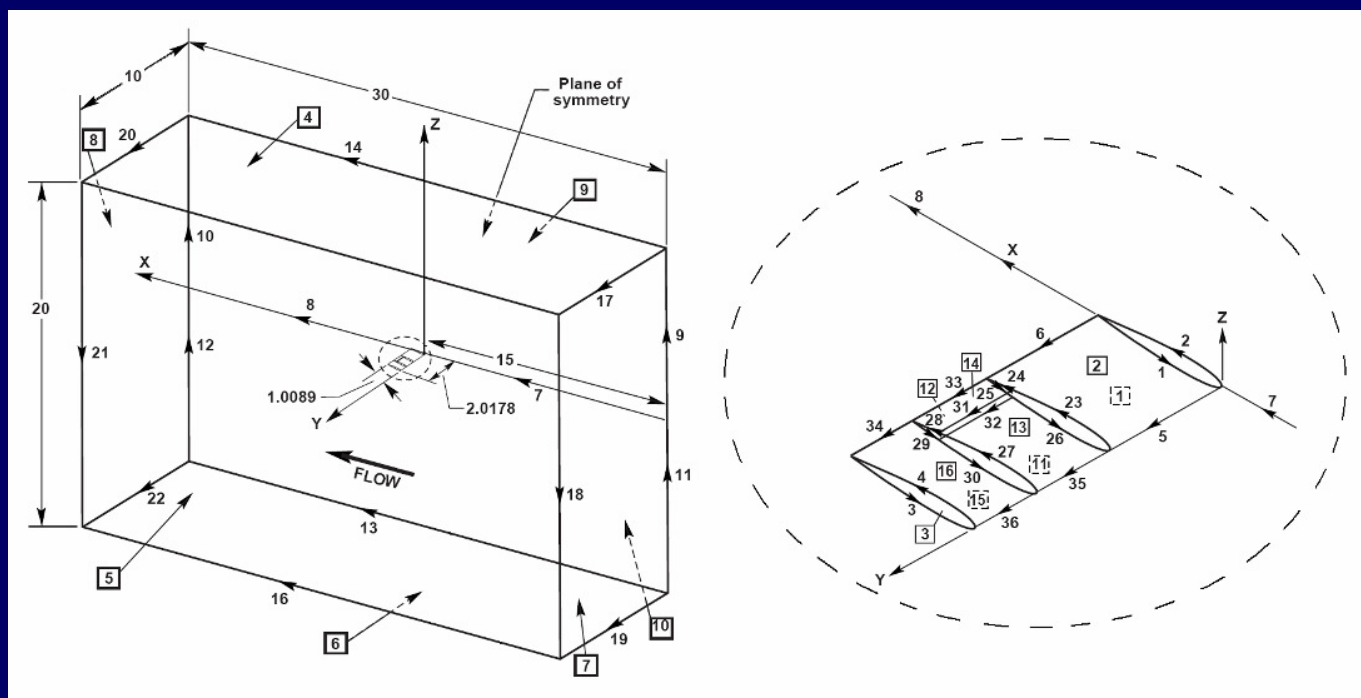

2. Structural (a) and aerodynamic (b) surface grid of wing

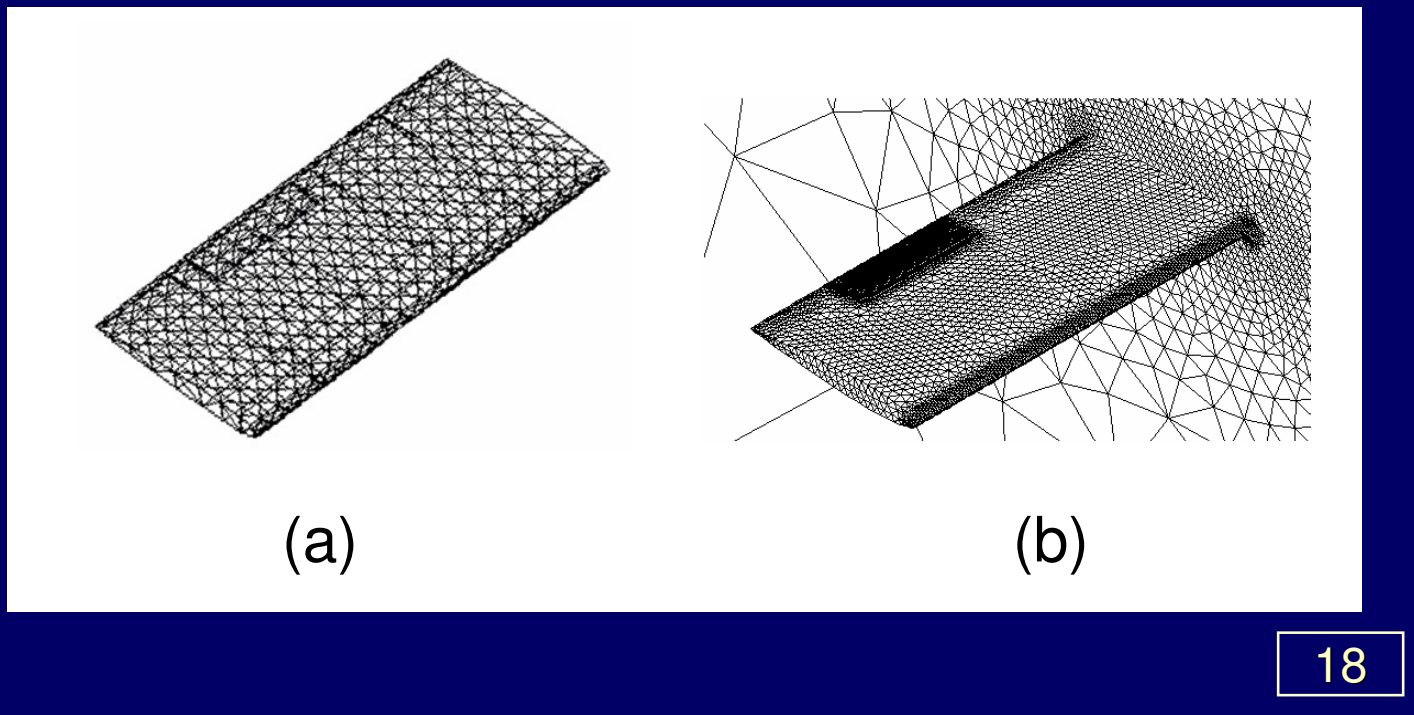




\section{Numerical Example II, 3-D (cont'd)}

- Structural modes of cantilever wing

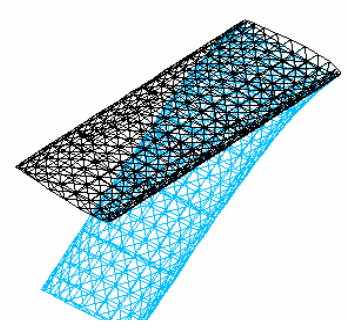

(a) mode 1,

3.521 Hz., 1B

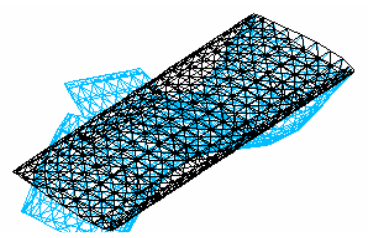

(e) mode 5 , 41.408 Hz., 3B

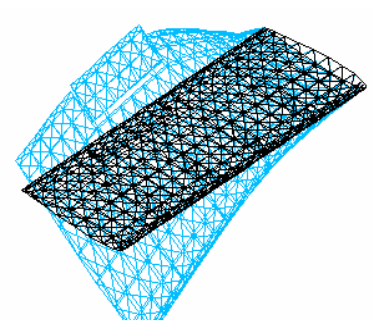

(b) mode 2, 14.819 Hz., $1 \mathrm{~T}$

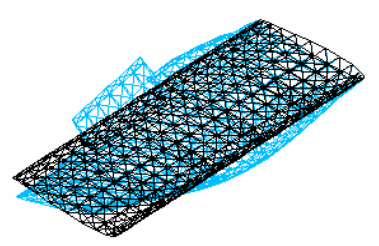

(f) mode 6 , 48.636 Hz., 2T

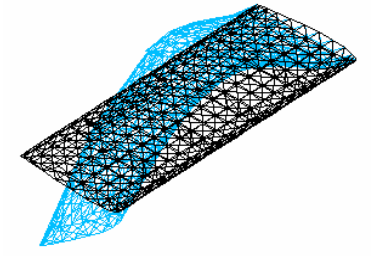

(c) mode 3 , 18.819 Hz., 2B

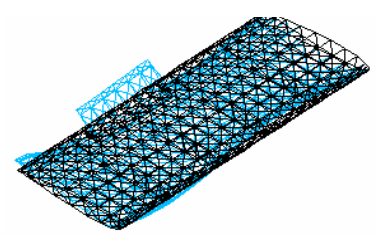

(g) mode 7, 59.797 Hz., 1B control

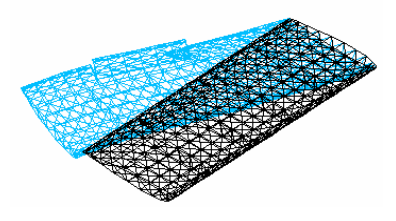

(d) mode 4, 38.026 Hz., in plane

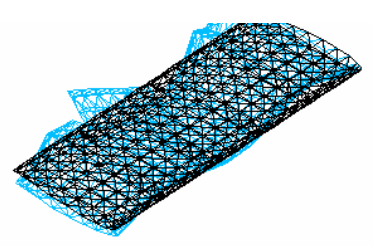

(h) mode 8, 64.958 Hz., 4B

- Aerodynamic steady-state pressure and Mach distribution $(M=2.0)$

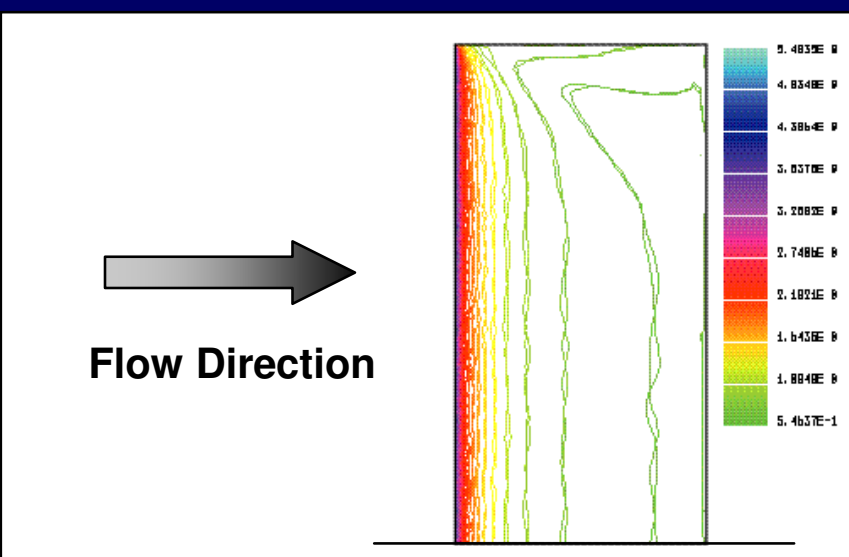

Pressure

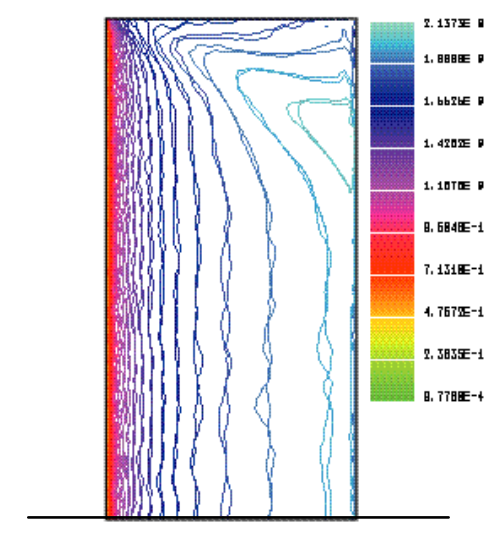

Mach 


\section{Numerical Example II, 3-D (cont'd)}

- Typical aeroelastic response plot (generalized displacement)
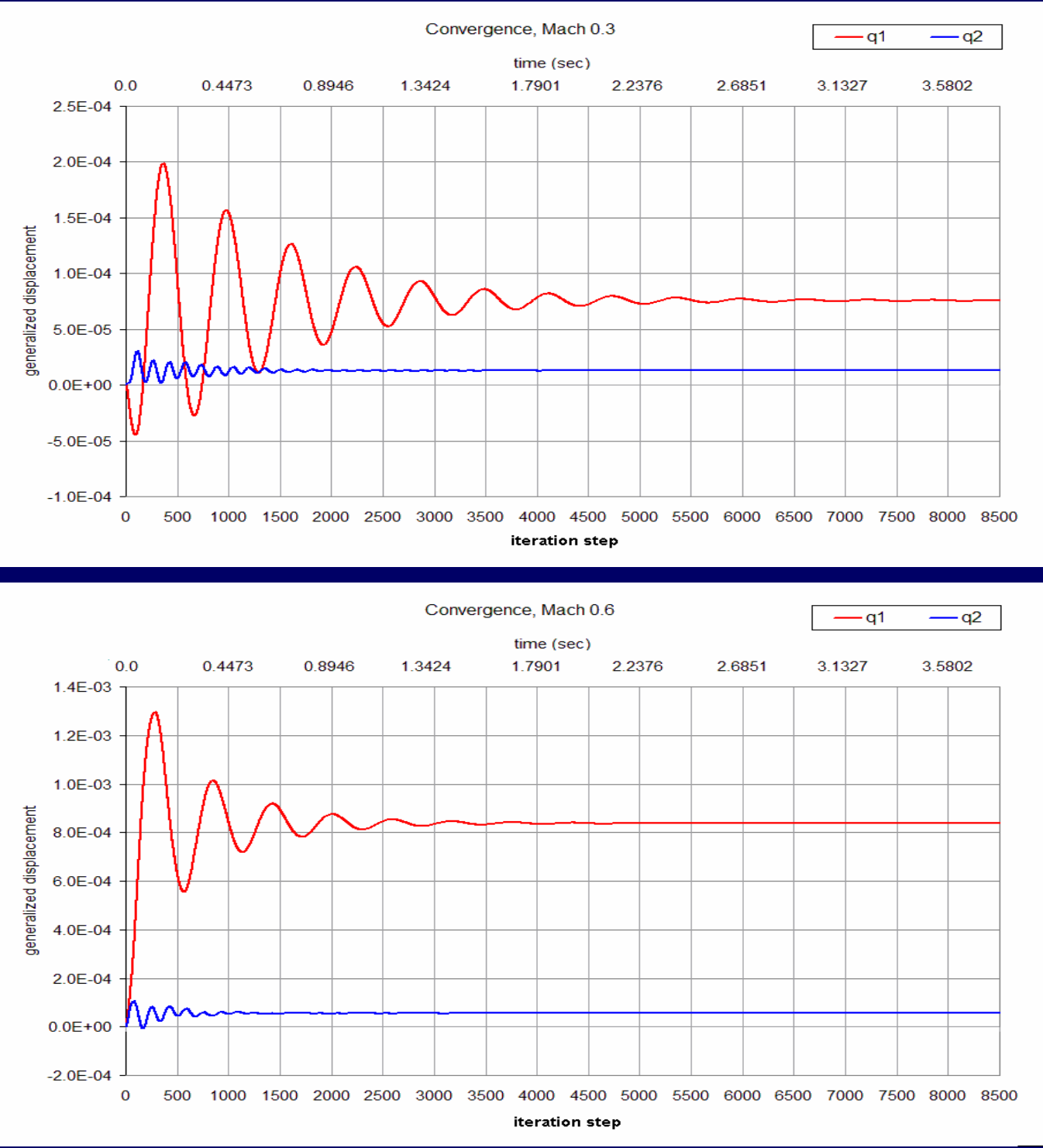


\section{Numerical Example II, 3-D (cont'd)}

- Layout of the acoustic computation sampling points

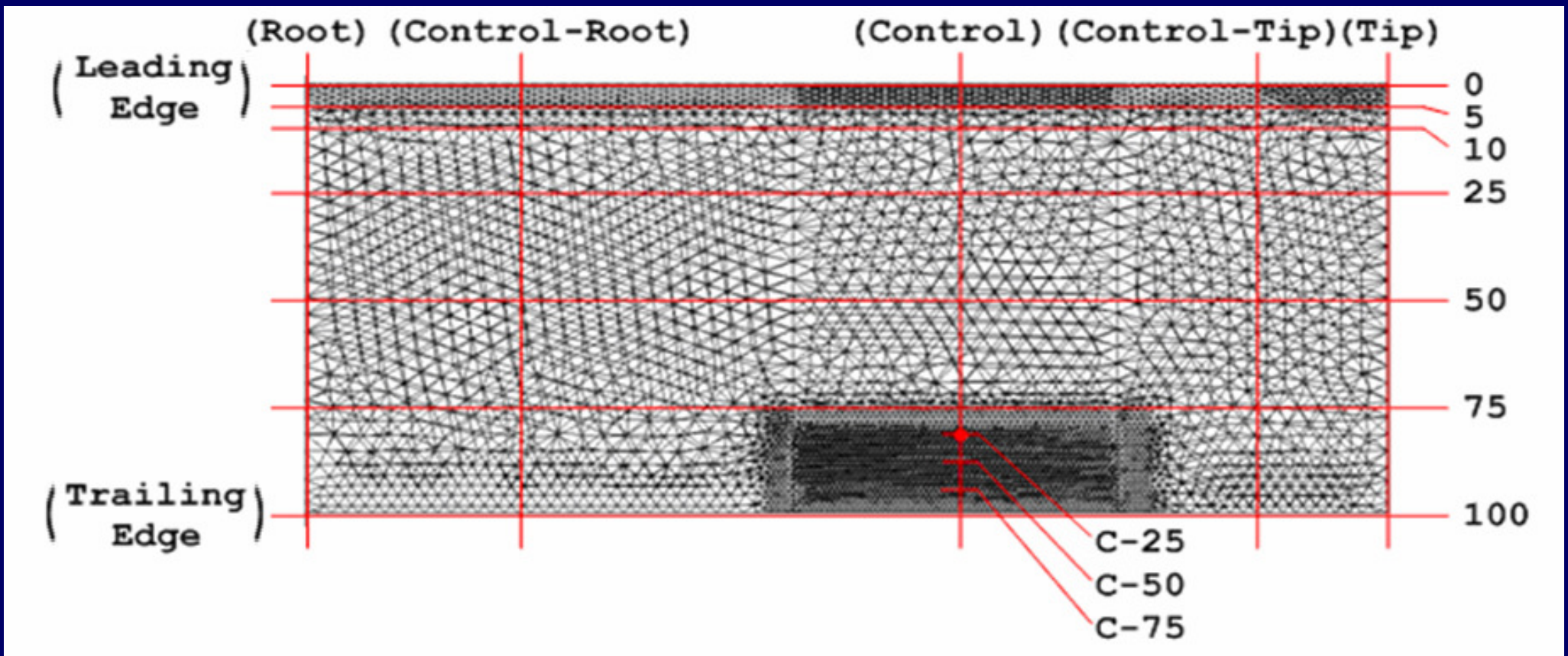




\section{Numerical Example II, 3-D (cont'd)}

- $\quad$ Mach 0.6

1. Aeroelastic unsteady pressure response (node, C25)

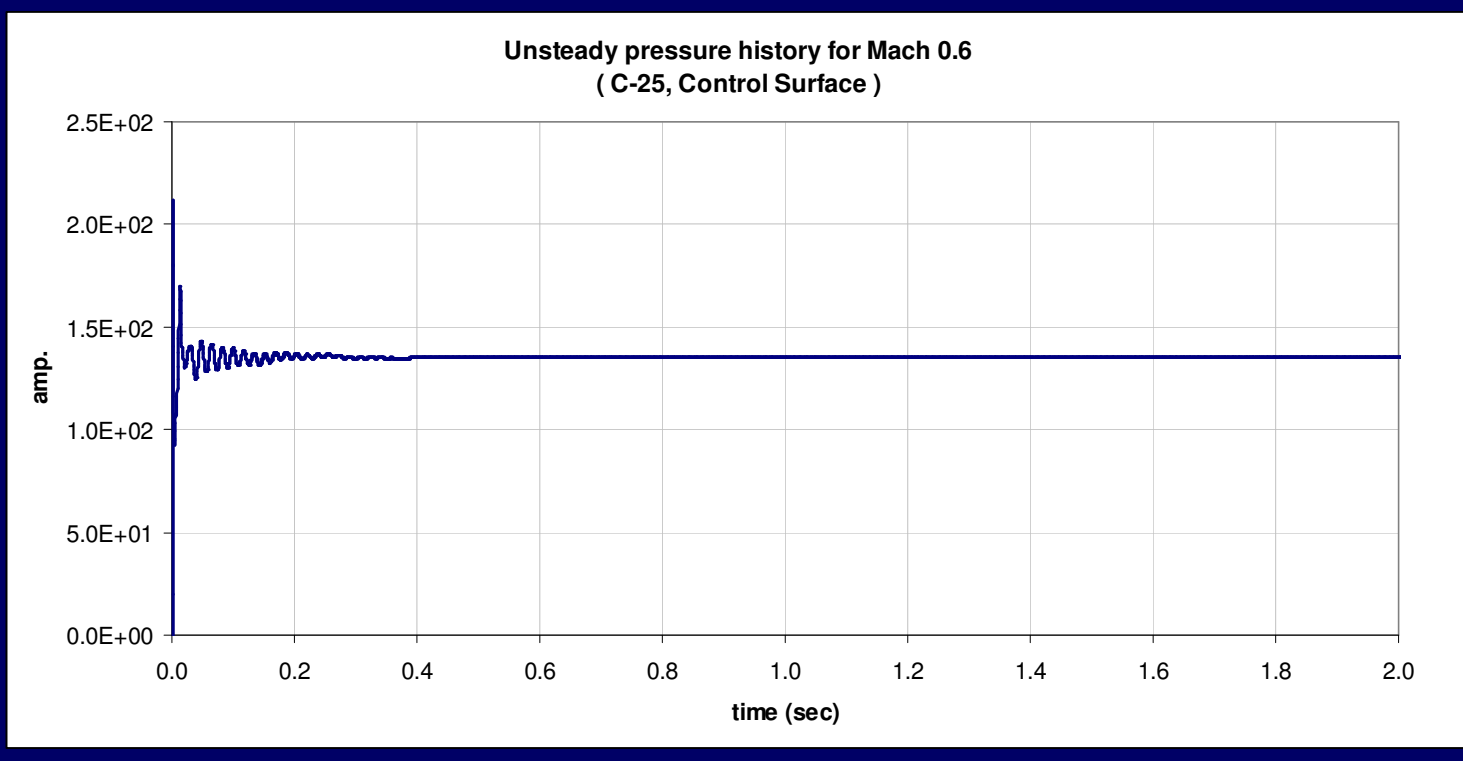

Acoustic wave frequencies for Mach 0.6 ( C-25, Control Surface)

2. Acoustic wave frequencies (node, C25)

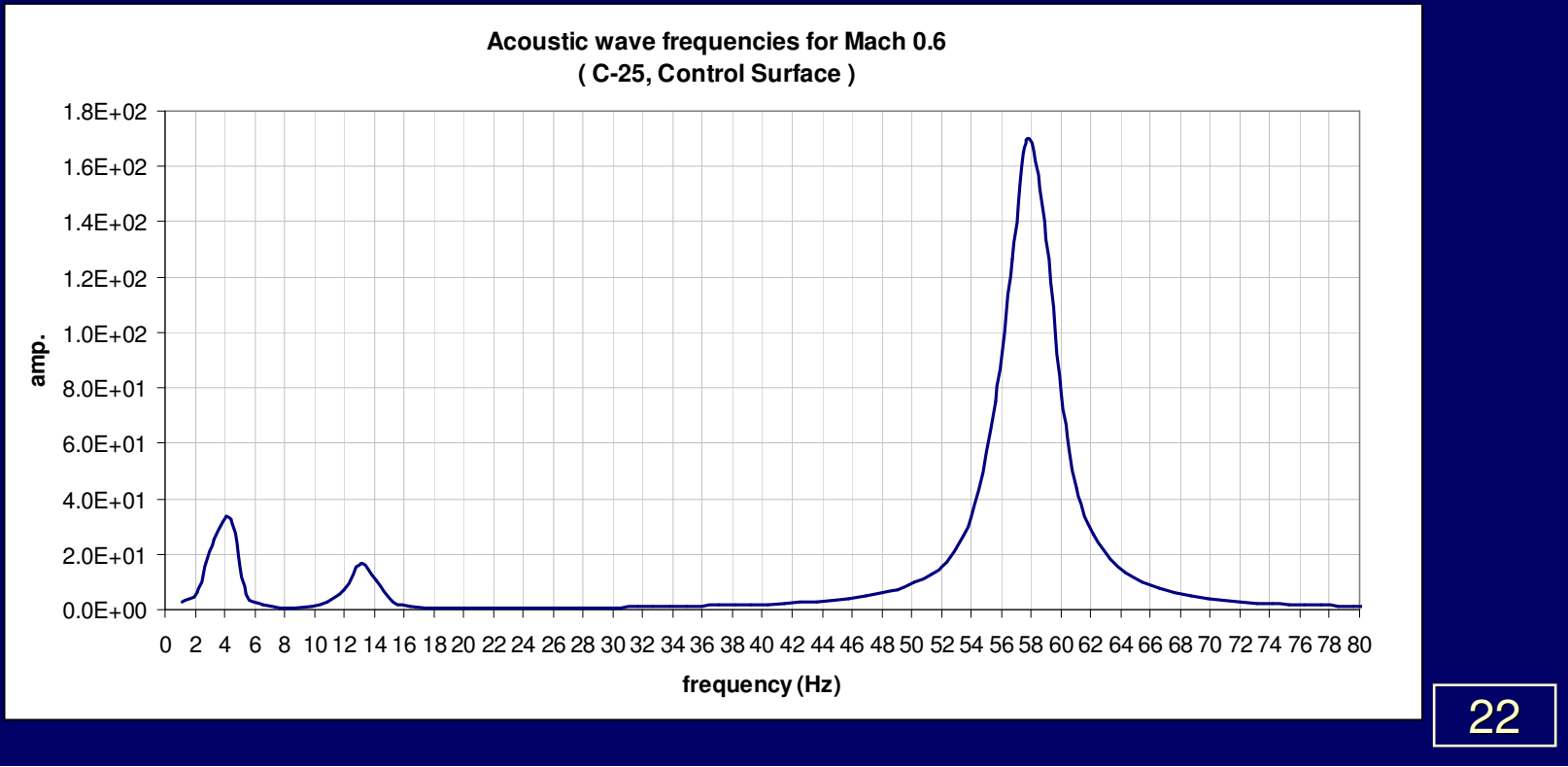




\section{Numerical Example II, 3-D (cont'd)}

- Sound Pressure Level (SPL) for a line along the cord length ( $20 \%$ from wing tip)

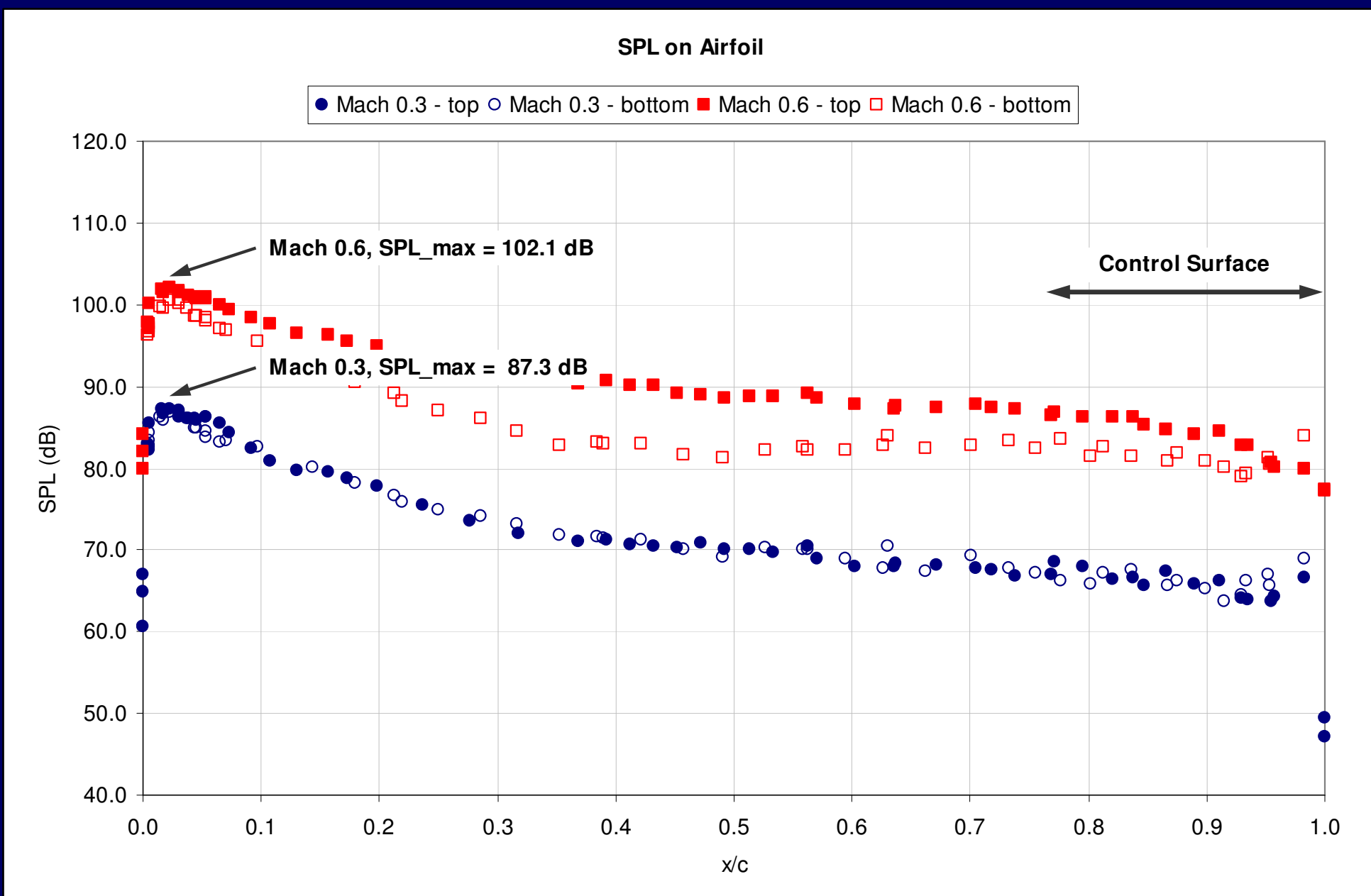




\section{Introductory remarks}

- Paper describes a numerical FE based aeroelastic-acoustics analysis methodology

- This is followed by solution details of a 2-D airfoil and results correlation with known theoretical solution

- Also presented the 3-D wing case and related results pertaining to vibration, steady and unsteady flow (CFD), aeroelastic and aeroelastic-acoustic simulations

- Further associated solution results are presented for a numerically simulated unsteady pressure data

- Also SPL results from a SOFIA flight sensor data is presented in some detail

- Provides a discussion on implementation of these techniques in an existing FE software suitable for solution of complex, practical problems

- Discussions and concluding remarks 


\section{Numerical Example III, simulated data}

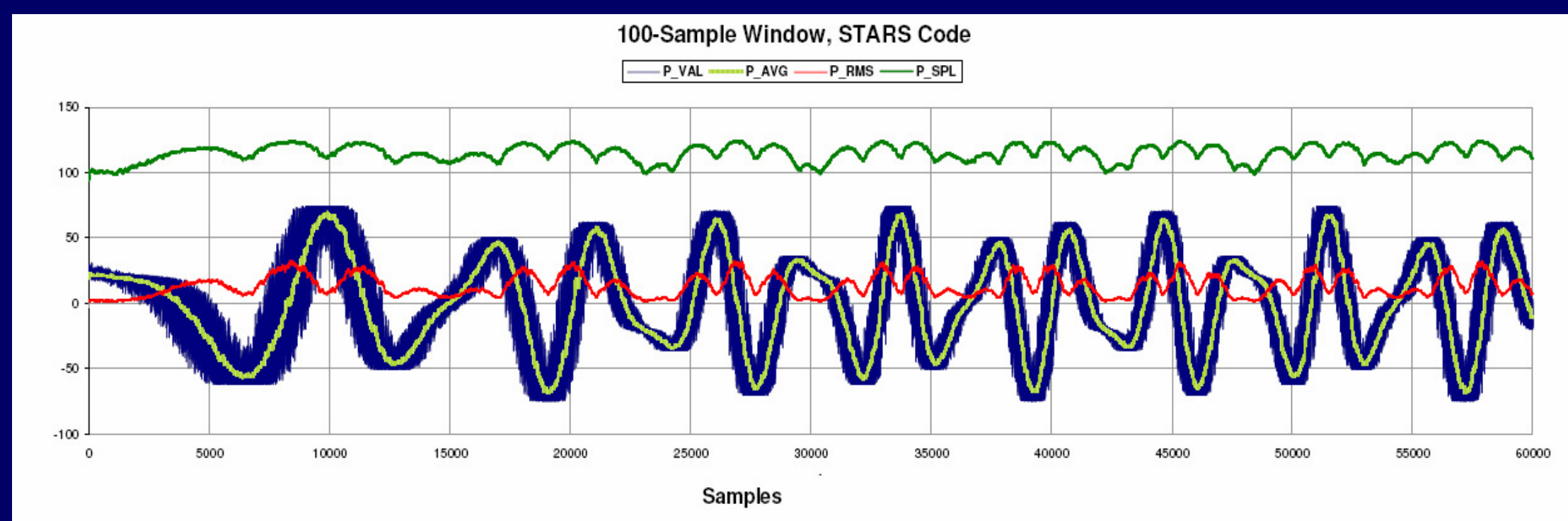

1000-Sample Window, STARS Code

-P_VAL -P_AVG_P_PMS -P_SPL

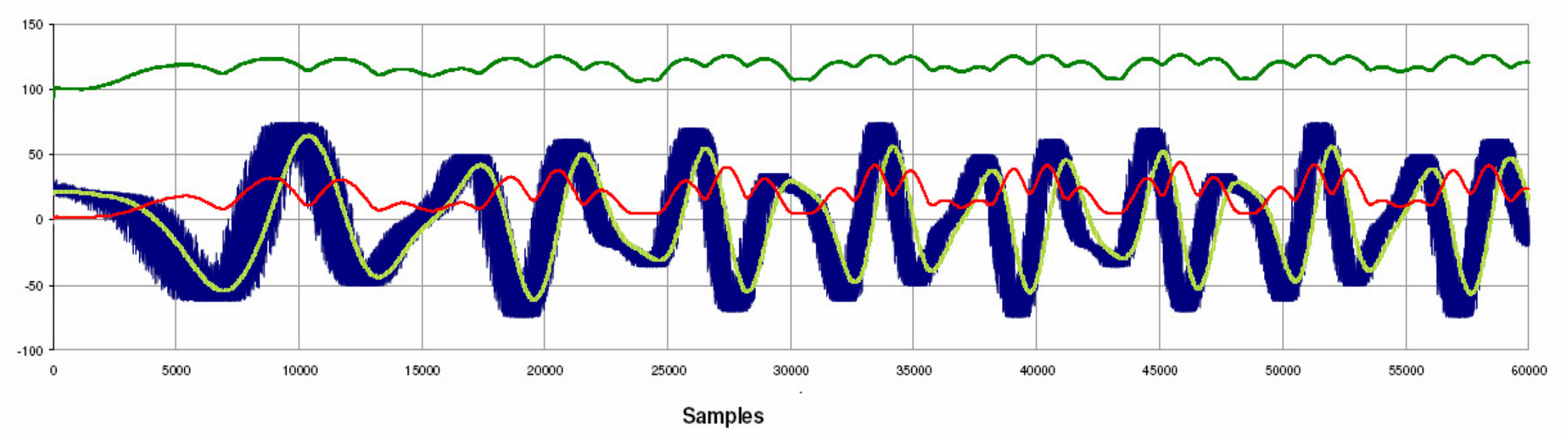




\section{Numerical Example III, simulated data (cont'd)}

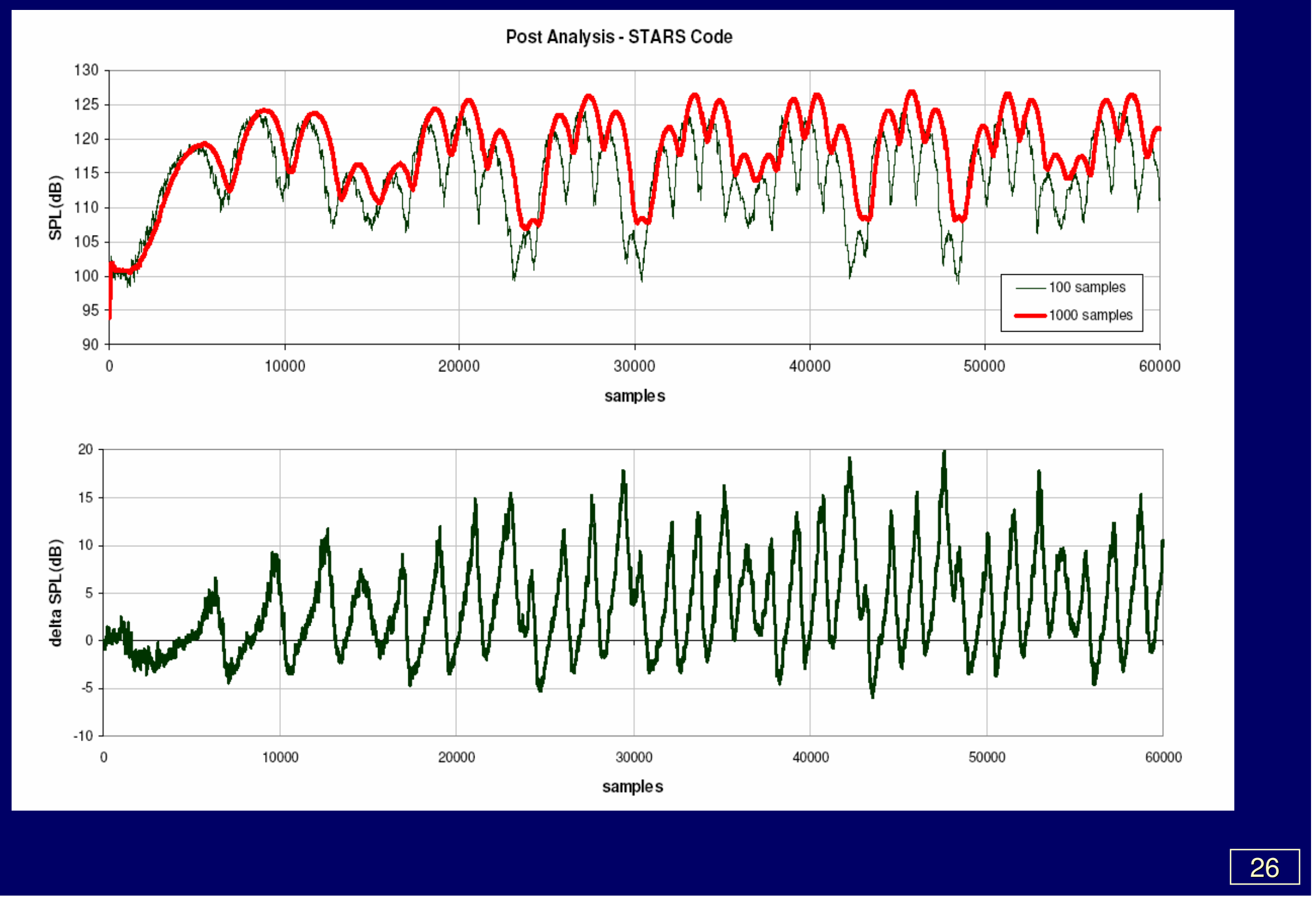




\section{Numerical Example III, simulated data (cont'd)}

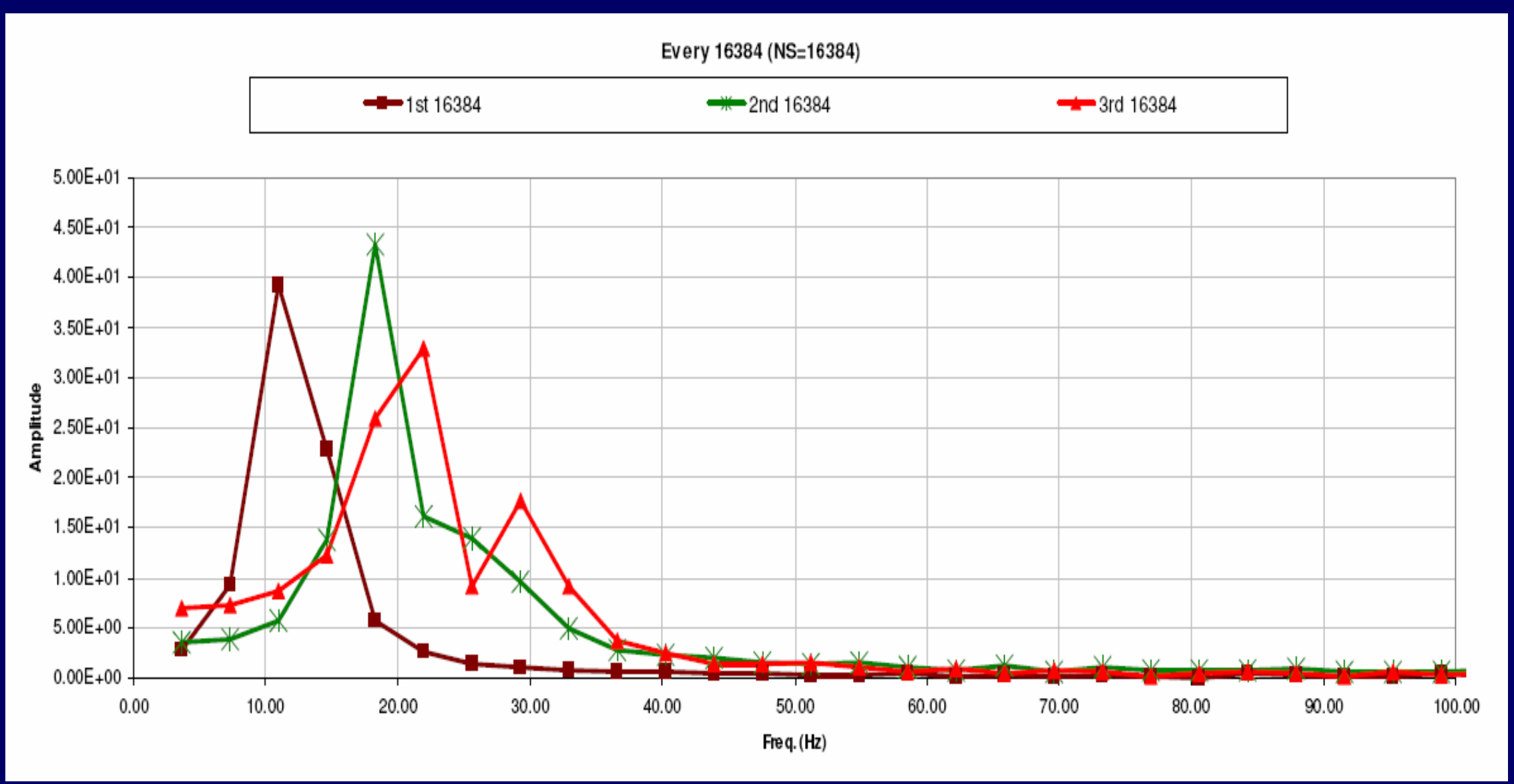




\section{Introductory remarks}

- Paper describes a numerical FE based aeroelastic-acoustics analysis methodology

- This is followed by solution details of a 2-D airfoil and results correlation with known theoretical solution

- Also presented the 3-D wing case and related results pertaining to vibration, steady and unsteady flow (CFD), aeroelastic and aeroelastic-acoustic simulations

- Further associated solution results are presented for a numerically simulated unsteady pressure data

- Also SPL results from a SOFIA flight sensor data is presented in some detail

- Provides a discussion on implementation of these techniques in an existing FE software suitable for solution of complex, practical problems

- Discussions and concluding remarks 


\section{Numerical Example IV, SOFIA measured unsteady pressure data}

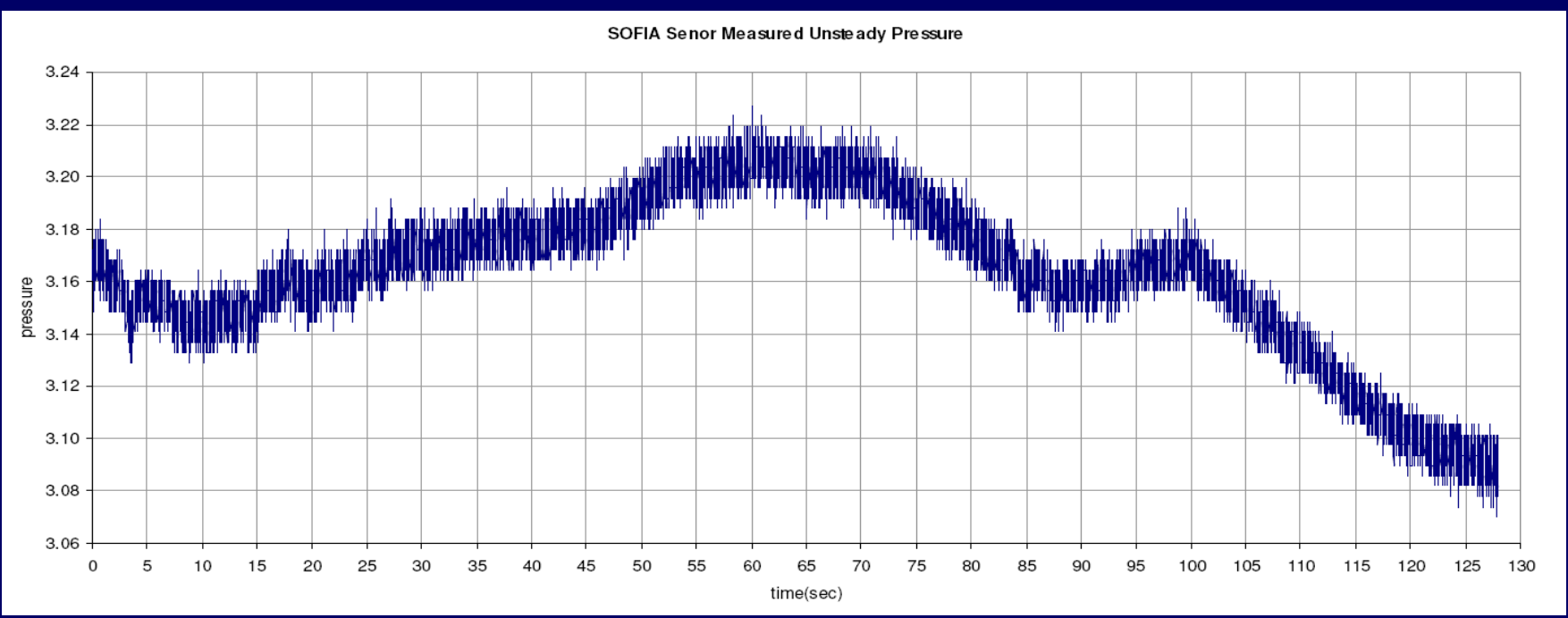

(a) Measured sensor data

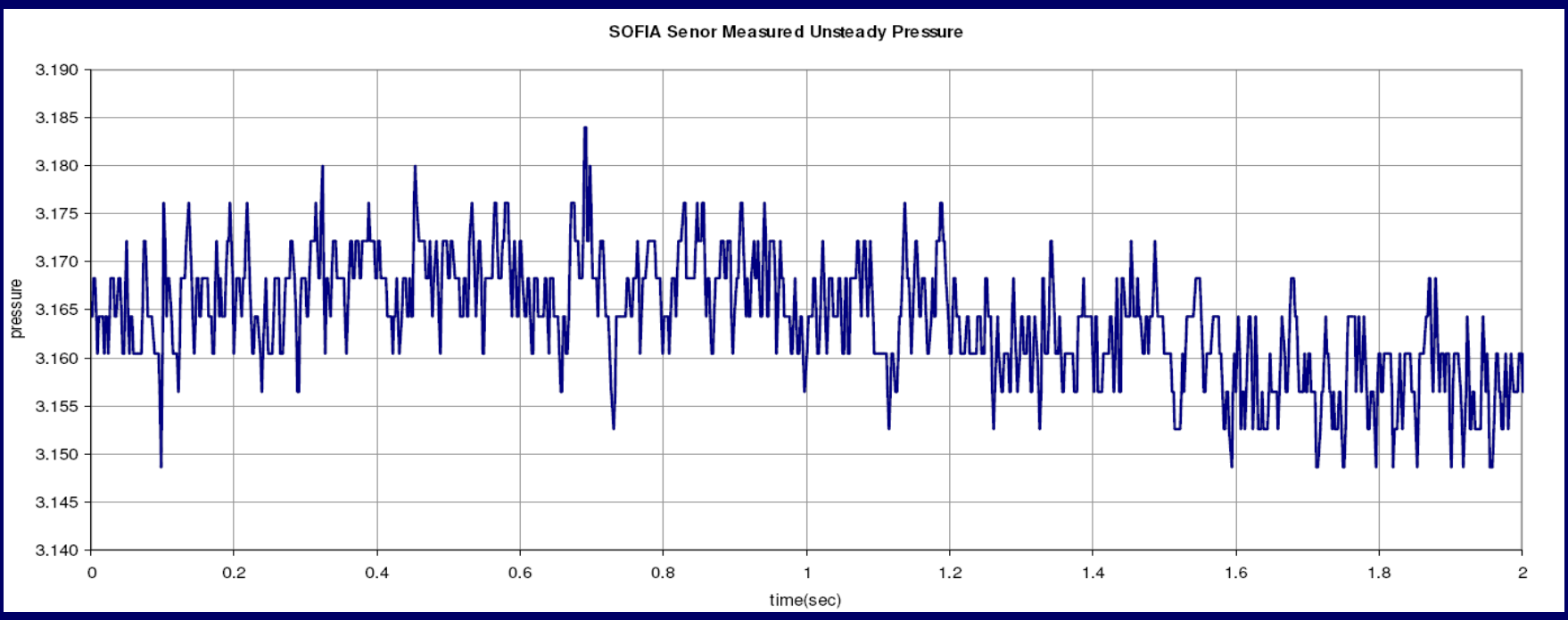

(b) Close up Measured sensor data

SOFIA sensor measured unsteady pressure data 


\section{Numerical Example IV, \\ SOFIA measured unsteady pressure data (cont'd)}

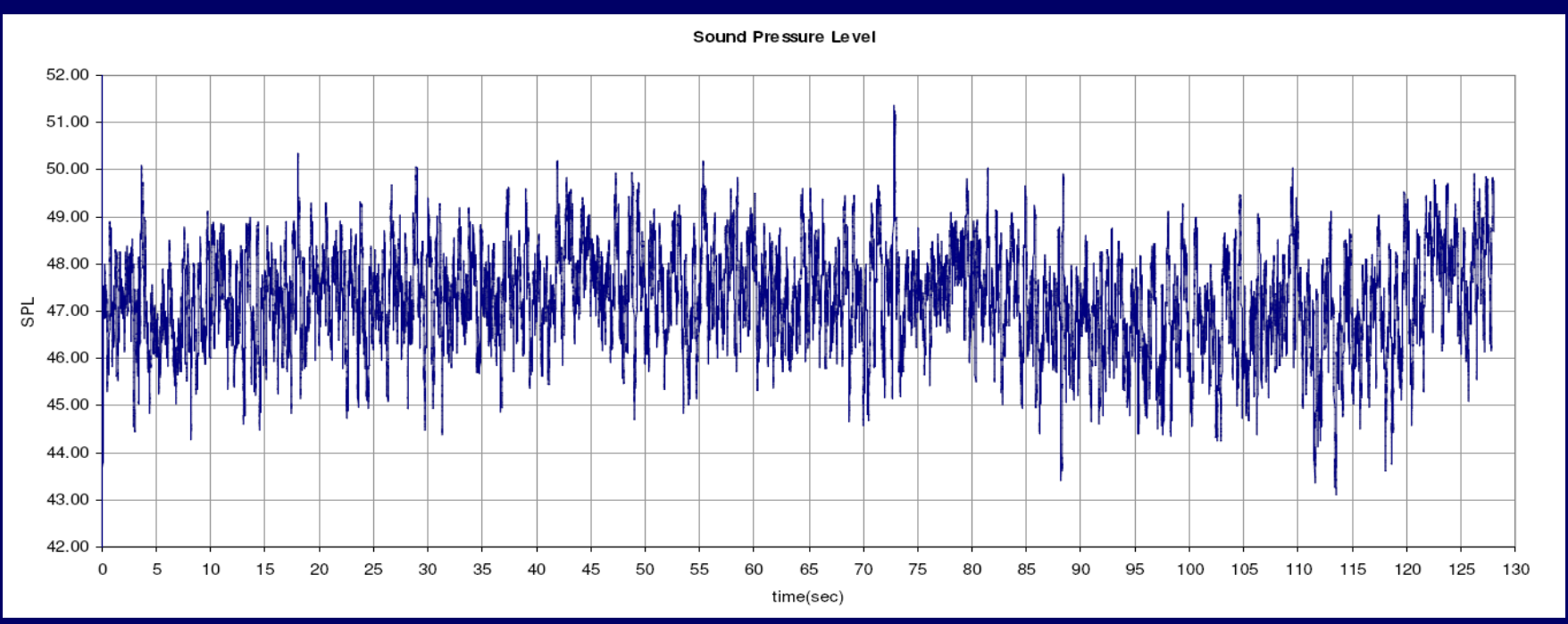

(a) SPL results data

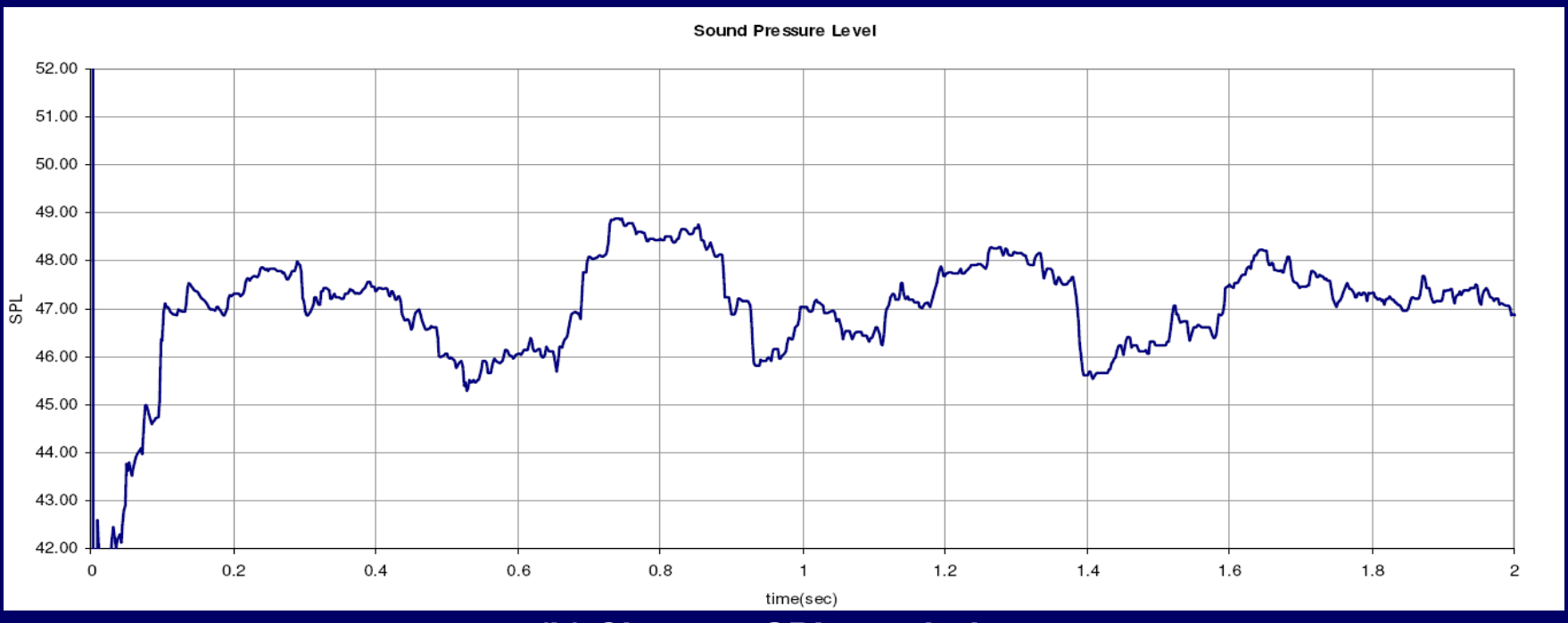

(b) Close up SPL result data

SOFIA calculated SPL results data 


\section{Numerical Example II, 3-D (additional)}

\section{- $\quad$ Mach 2.0}

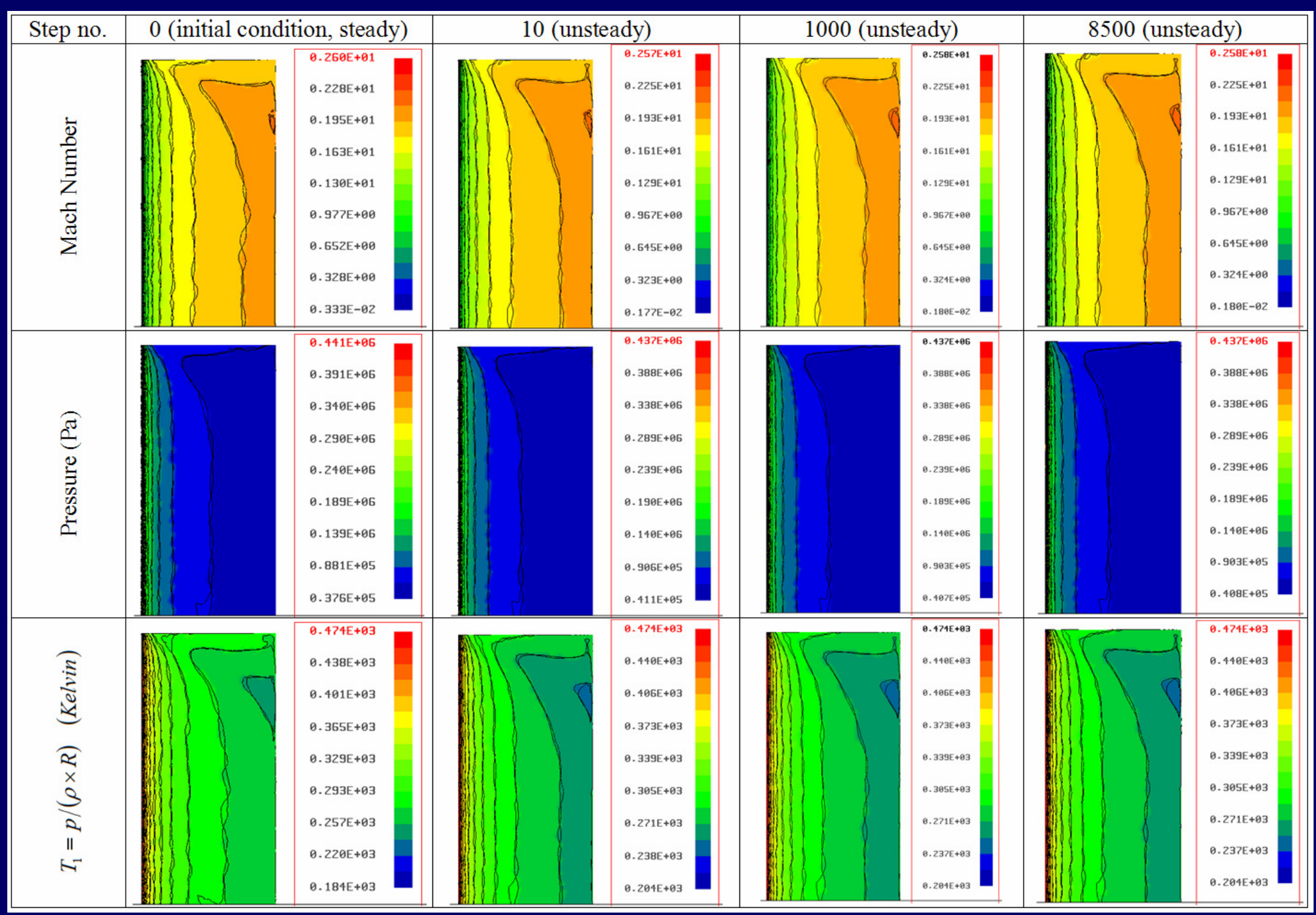




\section{Introductory remarks}

- Paper describes a numerical FE based aeroelastic-acoustics analysis methodology

- This is followed by solution details of a 2-D airfoil and results correlation with known theoretical solution

- Also presented the 3-D wing case and related results pertaining to vibration, steady and unsteady flow (CFD), aeroelastic and aeroelastic-acoustic simulations

- Further associated solution results are presented for a numerically simulated unsteady pressure data

- Also SPL results from a SOFIA flight sensor data is presented in some detail

- Provides a discussion on implementation of these techniques in an existing FE software suitable for solution of complex, practical problems

- Discussions and concluding remarks 


\section{STARS capability}

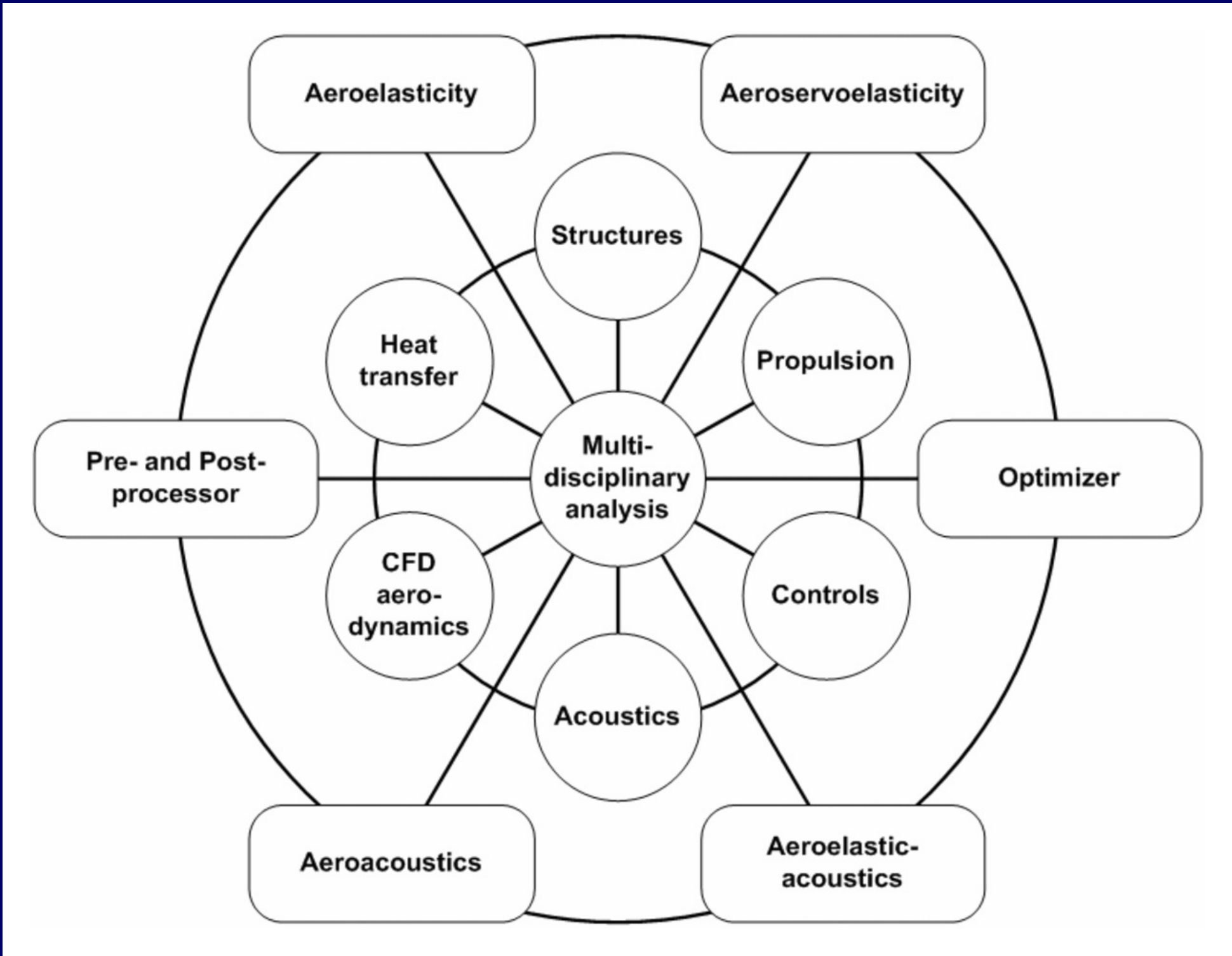




\section{Introductory remarks}

- Paper describes a numerical FE based aeroelastic-acoustics analysis methodology

- This is followed by solution details of a 2-D airfoil and results correlation with known theoretical solution

- Also presented the 3-D wing case and related results pertaining to vibration, steady and unsteady flow (CFD), aeroelastic and aeroelastic-acoustic simulations

- Further associated solution results are presented for a numerically simulated unsteady pressure data

- Also SPL results from a SOFIA flight sensor data is presented in some detail

- Provides a discussion on implementation of these techniques in an existing FE software suitable for solution of complex, practical problems

- Discussions and concluding remarks 


\section{Concluding Remarks}

- An integrated finite element based aeroelastic-acoustics analysis algorithm presented

- Its implementation in a code, suitable for large scale computation, is also presented in some detail

- Numerical verification example problem is demonstrated

- A 3-D wing problem is analyzed in detail, that demonstrate the FE codes capability to solve practical problems routinely

- Also presented a simulated example problem followed by a SOFIA flight measured data solution 


\section{Thank you!}

QUESTIONS ? 